\title{
Transmission Line Synthesis Approach to Extending the Bandwidth of LEDs for Visible Light Communication
}

\author{
Temitope Odedeyi, Paul Anthony Haigh and Izzat Darwazeh \\ Department of Electronic and Electrical Engineering, University College London \\ London, United Kingdom \\ \{temitope.odedeyi.13; p.haigh; i.darwazeh\}@ucl.ac.uk
}

\begin{abstract}
This paper proposes, for the first time, a transmission line synthesis approach to extending the bandwidth of lightemitting diodes (LEDs) in the context of high capacity visible light communications links. As opposed to the more traditional pre-distortion, amplitude equalisation or driver circuitry based approaches, the extension in bandwidth is achieved by incorporating the LED diffusion capacitance into a pseudo-artificial transmission line ( $p$-ATL) cell with significantly improved transmission and cut-off properties. With the proposed technique, we show the possibility of achieving close to $400 \%$ improvement in bandwidth with studies based on a verified LED equivalent model. It is envisaged that the proposed approach will enable bespoke driver circuits based on the individual characteristics of LEDs, while combination with existing bandwidth extension schemes can lead to further improvement.
\end{abstract}

Index Terms-Visible light communication; light emitting diodes; bandwidth extension; transmission line synthesis

\section{INTRODUCTION}

Visible light communications (VLC) is an emerging technology that has become the focus of enormous attention in recent years $[14,22,33]$. VLC operates on the principle of intensity modulation and direct detection of visible wavelengths using light-emitting diodes (LEDs) and silicon photodetectors, respectively. One of the most interesting applications for VLC is as an access network in a converged multi-technology $5 \mathrm{G}$ domain $[2,9,25,31]$, where existing radio-frequency technologies such as Wi-Fi can be complemented by high-speed, low latency optical signals. The key motivation for this is rooted in the impending capacity crunch, where it is envisaged that demand for data, particularly to users indoors, will vastly outstrip the available spectra. In [1], it is envisaged that $>30 \mathrm{~EB} / \mathrm{month}$ will be required to satisfy end users by 2020 and hence, new areas of the electromagnetic spectrum are being investigated for high-speed communications service provisioning. VLC is one of the most promising candidates due to its wide, license-free spectrum (380-780 nm), low capital expenditure and ability to provide simultaneous room illumination as well as data communications capabilities.

The main drawback to VLC, however, is the modulation bandwidth of LEDs, which is often limited to just several $\mathrm{MHz}[?, 12]$. In general, the high power LEDs used for room illumination are based on a black-emitting GaN semiconductor with a colour converting phosphor that spreads some of the energy to yellowish wavelengths, resulting in aggregated white light illumination. There are two fundamental processes that limit the overall device bandwidths; the carrier lifetime and the $R C$ time constant, connected to the diffusion capacitance of the diode [5]; and the slow temporal response of the colour converting phosphor [18]. Another key limitation of LEDs is their inherently non-linear electro-optic response, meaning transmitted signals must be carefully designed to avoid clipping or distortion [6].

To provide high bandwidths and subsequently, high capacity links, several methods have been proposed. These include (i) signal pre-distortion [15], (ii) post-distortion [17], (iii) amplitude equalization $[13,15]$, (iv) application of driver circuitry that reduces pulse decay time $[?, 28]$ and $(v)$ digital equalisation [12]. Techniques (i)-(iii) aim to simply reduce the capacitance of the device through high-pass filtering at different stages of the link. While these approaches have been demonstrated to support high transmission speeds, they are not generic and are normally designed on a trial-and-error basis. These methods are also often accompanied by a significant power penalty, as shown in Fig. 5 of [11], that directly impacts signal-to-noise ratio (SNR). On the other hand, in technique (v), the residual inter-symbol interference induced by out-ofband transmission is calculated and mitigated systematically, however high performance digital equalisers can be computationally expensive and are not always suitable for any given application.

In this paper, we propose an LED modulation-bandwidth extension technique based on distributed transmission line synthesis for the first time. The technique involves incorporating the LED diffusion capacitance into a pseudo-artificial transmission line (p-ATL) cell using series inductors and terminating with a resistor. The p-ATL may be designed to exhibit significantly improved transmission and cut-off properties. Importantly, as VLC evolves, there are numerous LED technologies emerging, such as $\mu$ LEDs [30], polymer LEDs (PLEDs) [10] and resonant cavity LEDs (RC-LEDs) [29], all of which have distinct characteristics. The proposed approach will enable bespoke driver circuits based on the individual characteristics of the LEDs, while combination with existing bandwidth extension schemes can lead to further improvement. 


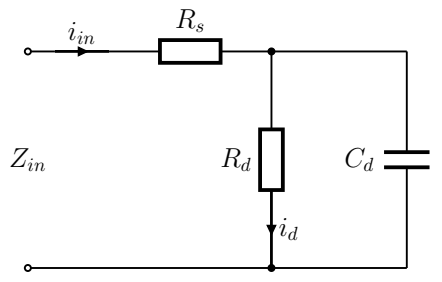

Fig. 1. A simplified LED small signal equivalent circuit.

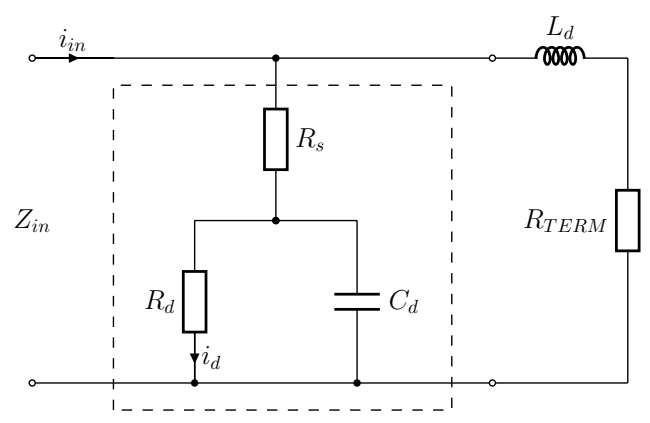

Fig. 2. LED equivalent circuit with $C_{d}$ incorporated into a p-ATL cell.

The outline of this paper is as follows: section II describes the proposed design concept and sets out the theoretical and analytical bases. In section III, the technique is demonstrated in simulation using an equivalent LED model reported in [5]. Section IV concludes the paper.

\section{DESIGN CONCEPT}

In Fig. 1 a single pole simplified equivalent circuit of an LED is shown [5,6] where $C_{d}$ is the diffusion capacitance, $R_{d}$ is the small signal dynamic (differential) resistance and $R_{s}$ accounts for the series resistance from the ohmic contact and device material [26]. From analysis of Fig 1, the input impedance $Z_{i n}$ of the LED is derived to be

$$
Z_{\text {in }}=R_{s}+\frac{R_{d}}{1+j \omega R_{d} C_{d}} .
$$

The LED exhibits low-pass filter properties, which are reflected in its equivalent circuit. At fixed bias, a reasonable approximation of the LED modulation bandwidth can be made based on its relation to $R C$ time constant $\tau$, ( $\tau$ corresponds to the LED recombination time constant $\tau_{c}$ [26]) as

$$
f_{c}=\frac{1}{2 \pi \tau_{c}}=\frac{1}{2 \pi C_{d} R_{d}} .
$$

The shunt capacitance $C_{d}$ maybe incorporated into a p-ATL cell using series inductance, with the aim of distributing the effect of the shunt capacitance more uniformly and across a wider passband. This approach to bandwidth extension is inspired by the techniques of distributed amplifier (DA) design [32], which have historically and recently led to record broadband amplifier bandwidths $[3,4,7,8]$. The design of the p-ATL cell follows the design of the input and output transmission lines of a single stage DA [19-21] and is based on the image parameter filter design method [16,24].

To achieve the desired distributed capacitance effect, an inductance $L_{d}$ is introduced at the input terminal of the LED (as shown in Fig. 2), based on the image impedance $\left(Z_{o}\right)$ relation for a lossless ATL, which is given by

$$
Z_{o}=\sqrt{\frac{L}{C}\left(1-\frac{\omega^{2} L C}{4}\right)}
$$

with the cut-off frequency of the ATL $\left(f_{c-A T L}\right)$ given by:

$$
f_{c-A T L}=\frac{\omega_{c}}{2 \pi}=\frac{1}{\pi \sqrt{L C}},
$$

where $L$ represents the series inductance required to achieve a distributed effect, $C$ is the shunt capacitance (which in our case corresponds to the LED diffusion capacitance $\left.C_{d}\right), \omega$ is the angular frequency and $\omega_{c}$ is the angular cut-off frequency $[23,32]$. From (3), the value of $L$ is conventionally chosen as the product of $C$ and the square of the DC value of $Z_{o}$ [32]. The terminating resistance $R_{T E R M}$ is conventionally chosen as the DC value of $Z_{o}$ to achieve a broadband match.

It may be observed from Fig. 2 that a current divider circuit is formed between the LED and the sum of $R_{T E R M}$ and the reactance of $L_{d}$, which reduces the effective LED drive current. Hence, as $R_{d}$ and $C_{d}$ are dependent on bias, the ATL needs to be designed at the parameters corresponding to the effective LED drive current.

However, for the proposed application, the lossless assumption commonly adopted in ATL synthesis is invalid due to the presence of $R_{d}$ and $R_{s}$. Furthermore, the parallel combination of $\left(R_{d}+R_{s}\right)$ and $R_{T E R M}$ also impacts $Z_{o}$, such that $R_{T E R M}$ does not provide an image impedance match even at low frequencies.

The input impedance of the LED with distributed input $Z_{i n}^{*}$, as shown in Fig. 2 is derived as

$$
Z_{\text {in }}^{*}=\left[R_{s}+\left(\frac{R_{d}}{1+j \omega R_{d} C_{d}}\right)\right] / /\left(j \omega L_{s}+R_{T E R M}\right),
$$

which on expansion becomes (6).

$$
Z_{\text {in }}^{*}=\frac{R_{T E R M}\left(R_{s}+R_{d}\right)+j \omega\left(R_{s} R_{d} R_{T E R M} C_{d}+L_{d}\left(R_{s}+R_{d}\right)\right)-\omega^{2} R_{s} R_{d} L_{d} C_{d}}{R_{T E R M}+\left(R_{s}+R_{d}\right)+j \omega\left(L d+R_{s} R_{d} C_{d}+R_{d} R_{T E R M} C_{d}\right)-\omega^{2} R_{d} C_{d} L_{d}} .
$$

There are two main observations from (5): firstly, the frequency response and cut-off behaviour of the LED is no longer solely dependent on the $R C$ time constant of the LED such that by appropriate choice of the p-ATL impedance $Z_{o}$ (with corresponding values of $L_{d}$ and $R_{T E R M}$ ), significant bandwidth improvement may be achieved. The second observation is that at $\mathrm{DC}, Z_{i n}^{*}=\left(R_{S}+R_{d}\right) / / R_{T E R M}$, which is lower than $Z_{\text {in }}$ in (1); such that while there is a gain in 
bandwidth, there is an associated loss in impedance magnitude.

The effect of the sacrifice of impedance magnitude for wider bandwidth on a VLC system would be a reduction in the optical power and output light intensity of the LED, such that while the modulation bandwidth improves, the power-bandwidth product does not improve commensurately. However, trading optical power for bandwidth is hardly a demerit in VLC, as these systems generally have low overall power consumption and high optical power outputs [26]. Moreover, considering Shannon's capacity equation $C=$ $B \log _{2}(1+\mathrm{SNR})$, it may be observed that while capacity increases linearly with increasing bandwidth, it only increases with the logarithm of the signal-to-noise ratio (SNR) [27]. Hence, the gain-bandwidth trade-off is beneficial insofar as the decrease in power output is not sufficiently large as to limit improvement in overall capacity.

\section{Performance Assessment}

To illustrate the effect of the p-ATL input technique so far described, Fig. 3 compares the input impedance of an LED with two cases in which the proposed technique is applied: $Z_{o}=Z_{i n}$, and $Z_{o}=Z_{i n} / 2$, assuming equal effective current drive LED parameters. LED equivalent model parameters derived and verified in [5] are employed. These parameters are presented in Table I with the values derived at $2 m A$ drive current selected for this study.

As reported in [5], the LED equivalent circuit model on its own has a predicted and verified 3 -db cut-off frequency of 18.7 MHz. In the first case, where the distributed transmission line is designed at $Z_{o}=Z_{i n}$, a threefold improvement in bandwidth is predicted, while the input impedance reduces by $\sim 50 \%$ which agrees with (5). In the second case (i.e. $Z_{o}=Z_{i n} / 2$ ), there is a further improvement in bandwidth of $65 \%$ which translates to $\sim 400 \%$ overall improvement in input impedance bandwidth compared to the LED performance at $66.7 \%$ overall loss in impedance magnitude.

To further demonstrate the utility of the proposed concept, we compare the response of the LED with the two application cases of the proposed technique as previously described (i.e. $Z_{o}=Z_{i n}$, and $Z_{o}=Z_{i n} / 2$ ) to a $100 \mathrm{MHz}$ modulating current. Again, the LED equivalent circuit parameters at $2 \mathrm{~mA}$ presented in [5] are adopted. Towards minimising the peaking effect observed in Fig 3, we also reduce the distributing inductance to $L_{d} / 2$ in both application cases. Results of this comparison is presented in Fig. 4.

From Fig. 4, we see that the current $i_{d}$ closely follows the profile of $Z_{i n}$ in both cases where the distributing inductance

Table I

LED MODEL PARAMETERS AT VARIOUS DRIVE CURRENTS [5]

\begin{tabular}{|c|c|c|c|}
\hline $\mathrm{Ib}(\mathrm{mA})$ & $R_{s}(\Omega)$ & $R_{d}(\Omega)$ & $C_{d}(\mathrm{nF})$ \\
\hline \hline 1 & 1.6 & 28.4 & 0.342 \\
\hline 2 & 1.6 & 14.4 & 0.59 \\
\hline 5 & 1.6 & 6.0 & 1.33 \\
\hline 10 & 1.6 & 3.0 & 2.67 \\
\hline
\end{tabular}

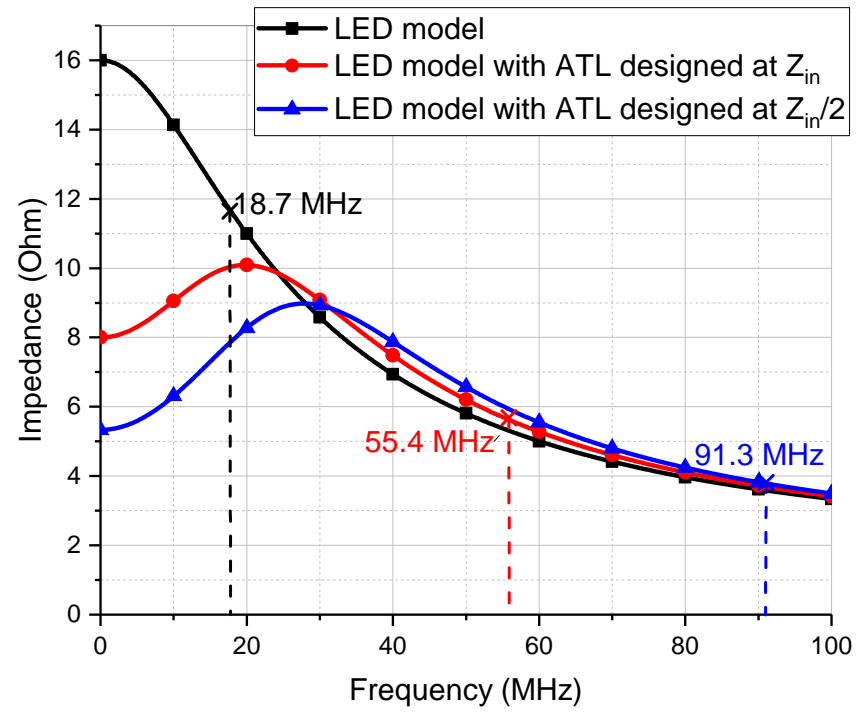

Fig. 3. Comparison of input impedance magnitude with the 3-dB bandwidth anotated.

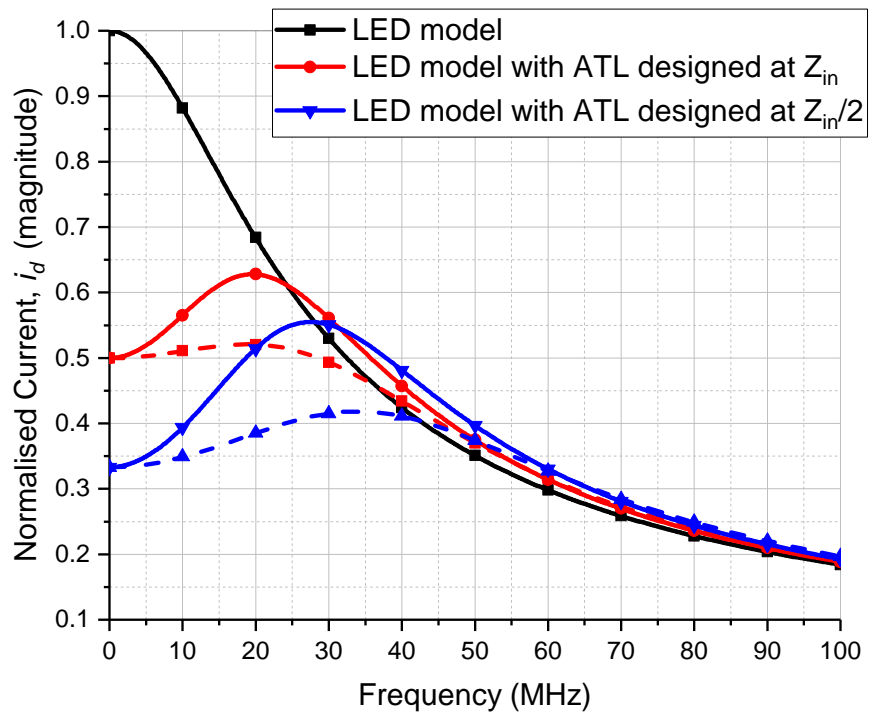

Fig. 4. Comparison of normalised current $i_{d}$ through the LED (dashed lines for inductance at $L_{d} / 2$ to reduce peaking).

is kept at $L_{d}$. In the cases in which distributing inductance of $L_{d} / 2$ is used, we notice a flatter gain profile, indication of a more uniform group delay and less signal distortion. This is significant, as by optimising the value of $L_{d}$ we may mitigate the challenge arising from the inherent non-linear modulation characteristics of LEDs as described in [6].

\section{CONCLUSION}

We have described an LED-bandwidth extension approach based on transmission line synthesis. The proposed technique involves incorporating the bandwidth limiting diffusion (shunt) capacitance of the LED into a pseudo-artificial transmission line ( $\mathrm{p}$-ATL) cell with series inductance and terminating with a resistor, based on the image impedance filter design method. 
The p-ATL can be designed for significantly improved cut-off, with $400 \%$ improvement presented in this paper, from studies based on a verified LED equivalent model. An added merit is the potential for achieving a flatter frequency response profile which correlates to a more uniform group delay and less signal distortion. It is expected that the proposed approach will enable bespoke driver circuits based on the individual characteristics of LEDs, while combination with existing bandwidth extension schemes can link to further improvement.

\section{ACKNOWLEDGEMENT}

The support of the Petroleum Technology Development Fund (PTDF) of Nigeria through the sponsorship of $\mathrm{T}$. Odedeyi's $\mathrm{PhD}$ program is acknowledged. This work was also supported by the UK EPSRC project EP/P006280/1: Multifunctional Polymer Light-Emitting Diodes with Visible Light Communications (MARVEL).

\section{REFERENCES}

[1] P. H. Pathak, X. Feng, P. Hu, and P. Mohapatra, "Visible light communication, networking, and sensing: A survey, potential and challenges," IEEE Communications Surveys Tutorials, vol. 17, no. 4, pp. 2047-2077, Fourthquarter 2015.

[2] S. Wu, H. Wang, and C.-H. Youn, "Visible light communications for 5G wireless networking systems: from fixed to mobile communications," Ieee Network, vol. 28, no. 6, pp. 41-45, 2014.

[3] D. Karunatilaka, F. Zafar, V. Kalavally, and R. Parthiban, "LED based indoor visible light communications: State of the art." IEEE communications surveys and tutorials, vol. 17, no. 3, pp. 1649-1678, 2015.

[4] M. Ayyash, H. Elgala, A. Khreishah, V. Jungnickel, T. Little, S. Shao, M. Rahaim, D. Schulz, J. Hilt, and R. Freund, "Coexistence of WiFi and $\mathrm{LiFi}$ toward 5G: concepts, opportunities, and challenges," IEEE Communications Magazine, vol. 54, no. 2, pp. 64-71, 2016.

[5] C.-X. Wang, F. Haider, X. Gao, X.-H. You, Y. Yang, D. Yuan, H. Aggoune, H. Haas, S. Fletcher, and E. Hepsaydir, "Cellular architecture and key technologies for $5 \mathrm{G}$ wireless communication networks," IEEE Communications Magazine, vol. 52, no. 2, pp. 122-130, 2014.

[6] M. B. Rahaim and T. D. Little, "Toward practical integration of dualuse VLC within 5G networks," IEEE Wireless Communications, vol. 22, no. 4, pp. 97-103, 2015.

[7] L. Feng, R. Q. Hu, J. Wang, P. Xu, and Y. Qian, "Applying VLC in 5G networks: Architectures and key technologies," IEEE Network, vol. 30, no. 6, pp. 77-83, 2016.

[8] [-], "Data traffic forecast update 2014-2019," Cisco Visual Network Index Global Mobile, 2015.

[9] P. A. Haigh, Z. Ghassemlooy, S. Rajbhandari, I. Papakonstantinou, and W. Popoola, "Visible light communications: $170 \mathrm{Mb} / \mathrm{s}$ using an artificial neural network equalizer in a low bandwidth white light configuration," Journal of lightwave technology, vol. 32, no. 9, pp. 1807-1813, 2014.

[10] W. Cheung, P. Edwards, and G. French, "Determination of LED equivalent circuits using network analyser measurements," in Optoelectronic and Microelectronic Materials Devices, 1998. Proceedings. 1998 Conference on. IEEE, 1999, pp. 232-235.

[11] S. Miyahara, S. Aono, and Y. Matsumoto, "Preproduction of LED driver for visible light communications and evaluation of response performance of visible LED," Technical report of IEICE, ICD2005-44, pp. 25-30, 2005.

[12] P. Deng, M. Kavehrad, and M. A. Kashani, "Nonlinear modulation characteristics of white LEDs in visible light communications," in Optical Fiber Communication Conference. Optical Society of America, 2015, pp. W2A-64.

[13] Y.-F. Liu, Y. C. Chang, C.-W. Chow, and C.-H. Yeh, "Equalization and pre-distorted schemes for increasing data rate in in-door visible light communication system," in Optical Fiber Communication Conference and Exposition (OFC/NFOEC), 2011 and the National Fiber Optic Engineers Conference. IEEE, 2011, pp. 1-3.
[14] H. L. Minh, D. O’Brien, G. Faulkner, L. Zeng, K. Lee, D. Jung, Y. Oh, and E. T. Won, "100-mb/s NRZ visible light communications using a postequalized white LED," IEEE Photonics Technology Letters, vol. 21, no. 15, pp. 1063-1065, Aug 2009.

[15] X. Huang, J. Shi, J. Li, Y. Wang, Y. Wang, and N. Chi, "750mbit/s visible light communications employing 64QAM-OFDM based on amplitude equalization circuit," in Optical Fiber Communication Conference. Optical Society of America, 2015, pp. Tu2G-1.

[16] H. Tanaka, Y. Umeda, and O. Takyu, "High-speed LED driver for visible light communications with drawing-out of remaining carrier," in Radio and Wireless Symposium (RWS), 2011 IEEE. IEEE, 2011, pp. 295-298.

[17] P. A. Haigh, Z. Ghassemlooy, H. Le Minh, S. Rajbhandari, F. Arca, S. F. Tedde, O. Hayden, and I. Papakonstantinou, "Exploiting equalization techniques for improving data rates in organic optoelectronic devices for visible light communications," Journal of Lightwave Technology, vol. 30, no. 19, pp. 3081-3088, 2012.

[18] D. Tsonev, H. Chun, S. Rajbhandari, J. J. McKendry, S. Videv, E. Gu, M. Haji, S. Watson, A. E. Kelly, G. Faulkner et al., "A 3-Gb/s singleLED OFDM-based wireless VLC link using a Gallium Nitride $\mu$ led," IEEE Photon. Technol. Lett., vol. 26, no. 7, pp. 637-640, 2014.

[19] P. A. Haigh, F. Bausi, H. Le Minh, I. Papakonstantinou, W. O. Popoola, A. Burton, and F. Cacialli, "Wavelength-multiplexed polymer LEDs: Towards $55 \mathrm{Mb} / \mathrm{s}$ organic visible light communications," IEEE Journal on Selected Areas in Communications, vol. 33, no. 9, pp. 1819-1828, 2015.

[20] C. L. Tsai and Z. F. Xu, "Line-of-sight visible light communications with InGaN-based resonant cavity LEDs," IEEE Photonics Technology Letters, vol. 25, no. 18, pp. 1793-1796, Sept 2013.

[21] E. F. Schubert, Light emitting diodes. Wiley Online Library, 2003 pp.60-61, 297-299.

[22] T. T. Wong, Fundamentals of distributed amplification. Artech House, 1993.

[23] Y. Baeyens, N. Weimann, V. Houtsma, J. Weiner, Y. Yang, J. Frackoviak, P. Roux, A. Tate, and Y. Chen, "Submicron InP D-HBT single-stage distributed amplifier with $17 \mathrm{~dB}$ gain and over $110 \mathrm{GHz}$ bandwidth," in Microwave Symposium Digest, 2006. IEEE MTT-S International. IEEE, 2006, pp. 818-821.

[24] A. Borjak, P. P. Monteiro, J. O’Reilly, and I. Darwazeh, "High-speed generalized distributed-amplifier-based transversal-filter topology for optical communication systems," IEEE Transactions on Microwave Theory and Techniques, vol. 45, no. 8, pp. 1453-1457, 1997.

[25] K. Eriksson, I. Darwazeh, and H. Zirath, "InP DHBT wideband amplifiers with up to $235 \mathrm{GHz}$ bandwidth," in 2014 IEEE MTT-S International Microwave Symposium (IMS2014). IEEE, 2014, pp. 1-4.

[26] — "InP DHBT distributed amplifiers with up to $235-\mathrm{GHz}$ bandwidth," IEEE Transactions on Microwave Theory and Techniques, vol. 63, no. 4, pp. 1334-1341, 2015.

[27] G. Nwaogu and C. Aitchison, "A very broadband microwave distributed amplifier using FETs," in 1981 11th European Microwave Conference, 1981, pp. 609-613.

[28] T. Odedeyi and I. Darwazeh, "Bandwidth enhancement technique for bipolar single stage distributed amplifier design," in 2017 IEEE Asia Pacific Microwave Conference (APMC). IEEE, Nov 2017, pp. 833836.

[29] $\_$, "Matrix single stage distributed amplifier design for ultra wideband application," in 24th IEEE International Conference on Electronics, Circuits and Systems (ICECS), Batumi, Georgia, 2017. IEEE, 2017.

[30] G. L. Matthaei, L. Young, and E. M. Jones, "Design of microwave filters, impedance-matching networks, and coupling structures. volume 2," DTIC Document, Tech. Rep., 1963, pp 85 - 86.

[31] D. M. Pozar, Microwave engineering. John Wiley \& Sons, 2009, pp $404-407$.

[32] C. Poole and I. Darwazeh, Microwave Active Circuit Analysis and Design. Academic Press, 2015, pp. 468-470.

[33] C. E. Shannon, "A mathematical theory of communication," Bell System Technical Journal, 1948. 